\title{
Einige Beobachtungen über die Flucht von adulten Lepidopteren aus den Netzen orbiteler Spinnen
}

\section{Journal Article}

Author(s):

Nyffeler, Martin; Benz, Georg

Publication date:

1981-08

Permanent link:

https://doi.org/10.3929/ethz-a-005800812

Rights / license:

In Copyright - Non-Commercial Use Permitted

Originally published in:

Anzeiger für Schädlingskunde, Pflanzenschutz, Umweltschutz 54(8), https://doi.org/10.1007/BF01905996 
Anz. Schädlingskde., Pflanzenschutz, Umweltschutz 54, 113-114 (1981)

(C) 1981, Verlag Paul Parey, Berlin und Hamburg

ISSN 0340-7330/InterCode: ASUMDT

\section{Einige Beobachtungen über die Flucht von adulten Lepidopteren aus den Netzen orbiteler Spinnen*}

Von M. NyFFElER und G. BenZ

Mit einer Tabelle

\begin{abstract}
Some observations on the escape of adult Lepidoptera from webs of orb-weaving spiders

Field studies near Zurich (Switzerland) have shown, that adult Lepidoptera often are able to escape from spider webs of the families Argiopidae and Tetragnathidae. In the food of these orb-weaving spiders Lepidoptera made up only a very small part. The capability of the lepidopterans to escape from spider webs seems to be of great ecological importance.
\end{abstract}

Lepidopteren dienen zahlreichen insektivoren Prädatoren als essentielle Nahrungsquelle und sind folglich einem starken Feinddruck ausgesetzt (vgl. ALCOCK, 1977). Neben Vögeln und Fledermäusen stellen die im Luftraum aufgespannten „Klebtröpfchen-Netze“ der Radnetzspinnen ein großes Gefahrenpotential für Lepidopteren-Imagines dar. $\mathrm{Da}$ in naturnahen Landschaften solche Netzspinnen in hohen Dichten auftreten können (Lohmeyer u. Pretscher, 1979; NyfreLER u. BENZ, 1981 a), besteht dort für jeden Falter eine große Wahrscheinlichkeit, während seiner Flüge mit Spinnennetzen in Berührung zu kommen.

Den amerikanischen Forschern EISNER et al. (1964) gelang es mittels origineller Experimente zu zeigen, $\mathrm{daß}$ die Schuppen der Lepidopteren eine wichtige Schutzfunktion gegen die "Klebtröpfchen-Netze" der Radnetzspinnen erfüllen. Sie beschrieben dies wie folgt: "Moths, by virtue of the loose scales that cover their wings and bodies, are admirably adapted to elude capture by orb-weaving spiders. Rather than sticking to the web, they may simply lose some of their scales to the viscid threads, and then fly on".

Im Rahmen von mehrjährigen nahrungsökologischen Studien in der Gegend von Zürich (Schweiz) beobachteten wir gelegentlich, daß in Spinnennetze hineingeflogene Lepidopteren sich aus den Netzen losreißen und unter Verlust einiger Flügelschuppen weiterfliegen konnten. Wenn sich Lepidopteren aus einem Radnetz freiflattern, entsteht jeweils ein Loch im Netz, an dessen Peripherie Flïgelschuppen kleben. Solche Löcher

* Ausgeführr mir Unterstïtzung durch den schweizerischen Nationalfonds zur Förderung der Wissenschaftlichen Forschung. fanden wir in den Radnetzen von Kreuzspinnen ( $A r$ giope bruennichi, Araneus diadematus, Araneus quadratus, Nuctenea cornuta, Singa nitidula, Meta segmentata) und Streckerspinnen (Tetragnatha extensa). Gelingt einem Falter die Flucht aus einem Spinnennetz, rettet er erstens sein Leben und beschädigt zusätzlich das Fangwerkzeug seines Feindes. Aus der beachtlichen Häufigkeit solcher durchlöcherter Radnetze schließen wir, daß einem großen Teil der mit Spinnennetzen in Berührung kommenden Lepidopteren die Flucht gelingt. Robinson und Robinson (1970) errechneten in Panama aus dem Lepidopteren-Anteil in der Spinnennahrung und aus der Zahl beschädigter Netze, daß $>50 \%$ der in Netze von Argiope argentata geratenen Falter entwischen konnten. Diese Beobachtungen aus der Schweiz und aus Panama zeigen, daß es den Lepidopteren gelingt, dem von Radnetzspinnen gegen Fluginsekten ausgeübten Prädatorendruck auszuweichen. Der von EisNer et al. (1964) entdeckte Antiprädator-Mechanismus scheint folglich unter Freilandbedingungen für die Lepidopteren eine wichtige Rolle zu spielen.

Diese Hypothese wird auch durch die Tatsache gestützt, daß Lepidopteren nur einen sehr geringen Anteil an der gesamten Nahrung vieler Radnetzspinnenarten ausmachen. In Tabelle 1 wird dies am Beispiel einer Radnetzspinnen-Gilde in einem Hochstaudenried bei Zürich-Opfikon gezeigt. Auch in zahlreichen anderen Biotopen bei Zürich betrug der LepidopterenAnteil in der Nahrung von Radnetzspinnen normalerweise $<1 \%$. Desgleichen konnten polnische Okologen in der Nahrung von Radnetzspinnen nur wenige Lepidopteren nachweisen (KAJAK, 1965; DabrowSKAProt u. Luczak, 1968). Da sich die meisten Spinnen, die ihre Netze in den Luftraum bauen, zur Hauptsache von kleinen Fluginsekten (v. a. Dipteren, Blattläuse) ernähren, sind sie auf Lepidopteren als Nahrungsquelle nicht angewiesen (vgl. NYFFELER u. BeNZ, 1981 b). Das Entwischen von Lepidopteren aus ihren Netzen dürfte daher für diese Spinnenarten an den meisten Standorten keine nahrungsökologische Bedeutung haben. Eine Ausnahme liegt eventuell bei den Argiope-Arten vor (vgl. Nyffeler u. Benz, $1981 \mathrm{c}$ ).

U. S. Copyright Clearance Center Code Statement: $0340-7330 / 81 / 5408-0113 \$ 02.50 / 0$ 


\begin{tabular}{|c|c|c|c|c|c|}
\hline Spinnenart & Mai & Juni & Juli & August & September \\
\hline bi & 一 & 一 & $一$ & $0 \%$ & $0,4 \%$ \\
\hline adematus & - & - & 一 & $0,6 \%$ & $0 \%$ \\
\hline adratus & - & - & $1,3 \%$ & $0 \%(708)$ & $0,1 \%(1140)$ \\
\hline$t a$ & $0 \%(141)$ & $0,2 \%$ & $0,4 \%(11$ & $0 \%(107)$ & - \\
\hline & - & - & - & $0 \%(471)$ & $0 \%(183)$ \\
\hline etragnatba extensa & $0 \%$ & $0 \%(331)$ & $0 \%(120)$ & - & - \\
\hline
\end{tabular}

Tabelle 1. Prozentualer Anteil Lepidopteren in der Nahrung von sechs Radnetzspinnenarten in einem Hochstaudenried bei Zürich-Opfikon (Mai bis September 1979)

Hinter dem Prozentsatz wurde in Klammern jeweils die Beuteanzahl, auf die sidh der Prozentsatz bezieht, angegeben

Außer den Lepidopteren gelingt es auch Skorpionsfliegen (Panorpa spp.) häufig aus Radnetzen zu entkommen (Thornhill, 1975). Die Fähigkeit von Lepidopteren und anderen Insekten aus Spinnennetzen auszubrechen, muß bei der Beurteilung der Radnetzspinnen als Schädlingsprädatoren in Forst- und AgroƠkosystemen in Rechnung gezogen werden.

\section{Zusammenfassung}

Beobachtungen bei Zürich ergaben, daß LepidopterenFalter oft in der Lage sind, sidh aus Spinnennetzen von Arten der Argiopidae und Tetragnathidae zu befreien. Lepidopteren machen nur einen sehr kleinen Teil der Nahrung dieser Spinnen aus. Diese Tatsache muß bei der Bewertung von Spinnen als Schädlingsfeinde mit berücksichtigt werden.

\section{Literaturverzeichnis}

Alcock, J., 1977: Animal Behavior: An Evolutionary Approach. Sinauer Associates, Inc., Massachusetts, $547 \mathrm{pp}$.

Dabrowska-Prot, E., and J. Luczak, 1968: Studies on the incidence of mosquitoes in the food of Tetragnatba montana Simon and its food activity in the natural habitat. Ekol. Pol. A 16, 843-853.

EisnliR, T., R. Alsop and P. Ettershank, 1964: Adhesiveness of spider silk. Science 146, 1058-1061.

KAJAK, A., 1965: An analysis of food relations between the spider - Araneus cornutus $\mathrm{Cl}$. and Araneus quadratus $\mathrm{Cl}$. - and their prey in meadows. Ekol. Pol. A $13,717-764$.

Lohmeyer, W., und P. Pretscher, 1979: Über das Zustandekommen halbruderaler Wildstauden-Quecken-Fluren auf Brachland in Bonn und ihre Bedeutung als Lebensraum für die Wespenspinne. Natur und Landschaft 54, 253-259.

Nyffeler, M., und G. Benz, 1981 a: Okologische Bedeutung der Spinnen als Insektenprädatoren in Wiesen und Getreidefeldern. Mitt. dtsch. Ges. allg. angew. Ent. (im Druck).

NyFFELER, M., und G. BenZ, 1981 b: Freilanduntersuchungen zur Nahrungsökologie der Spinnen: Beobachtungen aus der Region Zürich. Anz. Schädlingskde., Pflanzenschutz, Umweltschutz 54, 33-39.

NyfFeler, M., und G. Benz, $1981 \mathrm{c}$ : Eine Notiz zum Beutefangverhalten der Radnetzspinne Argiope bruennichi (Scopoli) (in Vorber.).

Robinson, M. H., and B. Robinson, 1970: Prey caught by a sample population of the spider Argiope argentata (Araneae: Araneidae) in Panama: a year's census data. Zool. J. Linn. Soc. 49, 345-358.

Thornhill, R., 1975: Scorpionflies as kleptoparasites of web-building spiders. Nature 258, 709-711.

Anschrift der Verfasser: Dipl.-Ing. Agr. Martin NypfELER und Prof. Dr. Grorg BeNZ, Entomologisches Institut, Eidg. Technische Hochschule, ETH-Zentrum, CH-8092 Zürich, Schweiz. 